ENTREVISTA 



\title{
DIÁLOGO ENTRE ULISES SCHMILL Y ROLANDO TAMAYO (ESCLARECIMIENTO DE ALGUNAS PERPLEJIDADES)
}

\author{
Ulises Schmill \\ Instituto Tecnológico Autónomo de México \\ Rolando Tamayo \\ Universidad Nacional Autónoma de México
}

\begin{abstract}
Nota de los editores: Como el lector puede ver, en este número de Doxa el lugar hasta ahora ocupado por la sección de entrevistas acoge un diálogo entre los filósofos del Derecho mejicanos Ulises SCHMILL y Rolando TAMAYO. La conversación «vertical» que implica el género de entrevista se ve así reemplazada por una conversación de tipo «horizontal», en la que los dos interlocutores se interrogan mutuamente y discuten sobre los aspectos que juzgan de mayor interés de sus respectivas obras y trayectorias. Nuestra intención es que en los próximos números de Doxa el género, más tradicional para nosotros, de la entrevista vaya alternándose, en proporciones que sólo la marcha de las cosas podrá determinar, con este nuevo género del diálogo.
\end{abstract}

Rolando Tamayo Salmorán: Ulises, sobre tu tesis de la provisionalidad del orden revolucionario, tengo la impresión que la expresión «provisional» no es del todo acertada. Entiendo muy bien que el «orden jurídico provisional» es un orden jurídico (quizás castrense o en estado de emergencia constante), pero un orden jurídico. El problema: ¿qué es provisional? La Academia de la Lengua señala que «provisional es algo que se hace, se haya o se tiene temporalmente». ¿Cuánto tiene que durar la beligerancia para que deje de ser «provisional»? Tengo la impresión de que en tu tesis de la existencia de un orden jurídico revolucionario, mientras persiste la lucha o la guerra revolucionaria, tiene carácter provisional; pero supongamos que los grupos beligerantes duran décadas ¿siguen siendo provisionales?

Ulises Schmill Ordóñez: No es correcta esta apreciación, pues no concuerda con los hechos históricos que he tomado en cuenta para formular mi tesis sobre la revolución. Como sabes, KELSEN analiza, correctamente, en mi opinión, el fenómeno de la revolución y determina el cambio jurídico que se opera con el triunfo de la guerra revolucionaria, relacionando todo esto con su postulado de la Norma Fundante Básica, como traduce VERNENGO la expresión alemana Grundnorm. 
El problema que yo me he planteado y he intentado resolver jurídicamente, cuál es la naturaleza jurídica de la guerra revolucionaria, mientras la lucha armada está desarrollándose. Este tema no ha sido suficientemente analizado y he llegado a la conclusión de que una revolución, antes de que triunfe en un territorio determinado sobre una población específica, puede interpretarse como un orden jurídico, cuyos ámbitos de validez son variables, dependiendo de las plazas sobre las cuales ejerce dominio o de las personas que se encuentran sometidas a ese orden. Que los ámbitos de validez del orden revolucionario sean variables, puede pero no tiene que ser provisional.

Si por esta provisionalidad se entiende que puede dar origen a un orden jurídico claramente establecido con sus ámbitos de validez fijos y determinados, sólo en este sentido pudiera afirmarse que es provisional. Pero debe observarse que generalmente el orden jurídico revolucionario, de manera general, constituye el fundamento de validez del orden jurídico creado por los órganos del propio orden revolucionario o por los que se establecen con base en sus normas.

Esto es lo que aconteció, por ejemplo, con la revolución mexicana de 1913. Considero que el Decreto 1491 de la legislatura del Estado de Coahuila y el Plan de Guadalupe de Venustiano Carranza son la Constitución política del orden revolucionario, pues sus estipulaciones claramente pueden verse como normas jurídicas, que establecen los órganos del orden. Durante la guerra revolucionaria estos órganos dictan normas, emiten moneda, sancionan a los que realizan los hechos que son considerados delictivos, etc. Hay una multiplicidad de prescripciones que los órganos del movimiento revolucionario dictan y, en el caso de México, el propio Plan de Guadalupe fue reformado para convocar a un Congreso Constituyente. Estas reformas constituyen las normas positivas que fundamentan el establecimiento del Congreso Constituyente de 1916-1917 y, por lo tanto, son el fundamento de validez de la Constitución actualmente en vigor en nuestro país.

R.T.S.: Generalmente a un movimiento revolucionario es considerado un ilícito dentro de un orden jurídico. ¿Siempre es así?

U.S.O.: Puedo contestar tu pregunta de la siguiente manera: un hecho histórico puede ser analizado desde distintos puntos de vista o perspectivas teóricas: sociológico, económico, político, incluso biológico (como en el libro de Vernon REYNOLDS y Ralph TANNER La Biología de la Religión) y, claro está, también jurídico. Cada una de estas perspectivas, tomando en cuenta la determinación que cualquiera de ellas haga de un cierto fenómeno o hecho histórico, lo aplica o lo proyecta sobre el mismo, determinando las propiedades que su punto de vista considera interesantes o pertinentes y determina las consecuencias o propiedades que se pueden derivar de sus tesis fundamentales. El movimiento revolucionario lo podemos comprender como un fenómeno político o de poder, pero es posible aplicarle los conceptos fundamentales de la jurisprudencia, que se utilizan para describir los órdenes jurídicos establecidos o evolucionados sometidos a su consideración. No hay razón alguna que justifique la no aplicación de los conceptos jurídicos a un orden revolucionario. Lo contrario es lo que hace el anarquismo con un orden jurídico plenamente formado: se rehúsa a aplicar los conceptos de la jurisprudencia a ciertos hechos históricos.

R.T.S.: Tengo la idea de que formalizas el concepto jurídico de la revolución. 
U.S.O.: Es muy exagerado decir que he formalizado el concepto jurídico de la revolución. He presentado un breve esquema de los enunciados jurídicos que describen un derecho positivo establecido y los que han de formularse referentes al orden revolucionario. Si quieres exponer en un enunciado jurisprudencial el sentido del orden jurídico o el significado del hecho de que un cierto hombre se encuentre en prisión un determinado número de años, tienes que partir, por lo menos, de la Constitución positiva de un orden jurídico. El enunciado jurisprudencial diría más o menos lo siguiente: supuesta la existencia de la Constitución positiva y que conforme a ella se llevaron a cabo diversos procesos legislativos estableciéndose el Código Penal y el Código de Procedimientos Penales y conforme a estas leyes se llevó a cabo un proceso jurisdiccional que culminó en la emisión de una sentencia condenatoria a un sujeto $\mathrm{X}$, puede afirmar jurídicamente que $\mathrm{X}$ debe estar varios años en prisión en cumplimiento de esa sentencia. Y así con los innumerables hechos de la experiencia jurídica. Debe observarse que los primeros eslabones de esta cadena normativa son constantes en la formulación de las reglas jurisprudenciales que dotan de sentido a los actos de la experiencia jurídica. Tengo entendido que tú tienes una concepción similar, cuyo origen, hay que decirlo, está en la teoría de KELSEN, en la parte que denomina «Nomodinámica». Pues bien, los enunciados jurisprudenciales que describen normativamente el significado de los actos que tienen lugar dentro del movimiento revolucionario, carecen de esos primeros eslabones de la cadena expuesta más arriba, pues no pueden referirse a la Constitución del Estado ni a sus leyes, sino que las cadenas normativas tienen, en las primeras posiciones, eslabones distintos y muchas veces dichas cadenas normativas son muy cortas en longitud. Ejemplo: supuesta la Constitución del orden revolucionario, como dijimos más arriba respecto del Plan de Guadalupe, y la emisión de disposiciones penales por el órgano establecido y el proceso y juicio de un sujeto, se dicta una sentencia de muerte al sujeto de marras y se le fusila ante un paredón.

R.T.S.: Ulises, en la Reconstrucción Pragmática de la Teoría del Derecho ${ }^{1}$ introduces en tu texto, que es muy claro, un sinnúmero de fórmulas que creo perturban y oscurecen el argumento.

U.S.O.: Como te dije en una ocasión, no son fórmulas en el sentido matemático de la expresión; son esquemas en los que las palabras que ocurren en los enunciados normativos, fundamentalmente los nombres, han sido sustituidas por letras u otros signos, para un fácil manejo y referencia, siendo un modo muy económico de presentar una pluralidad complicada de conceptos y establecer sus relaciones recíprocas de manera perspicua.

R.T.S.: Me parece que tu manejo del concepto de derogación, si no es contradictorio me parece, al menos, paradójico. Creo que nos debes un esclarecimiento de tu concepto de derogación y explicarnos qué relación tiene todo lo anterior con la derogación.

U.S.O.: Ésta es una pregunta muy importante y sutil. Pareciera que una revolución derogara el Derecho o el Estado contra el que se dirige, pues lo sustituye y el Derecho anterior deja de estar en vigor, pierde su validez, de manera similar a como una norma pierde su validez, es decir, deja de existir, por virtud de la derogación. Sin embargo, es-

${ }^{1}$ México: Editorial Themis, 1997 (Colección Teoría del Derecho). 
tamos en presencia de dos hechos jurídicos totalmente distintos. Déjame exponer primero mi concepto de la derogación. KELSEN tiene una teoría sobre la derogación que para mí no es aceptable. Dice que en la derogación nos encontramos con dos normas: la derogada y la derogante. Mientras que en la primera se establece un deber ser, en la segunda se establece un no deber ser. Considerando de modo estricto este concepto simplemente carece de sentido, pues si tomamos el «deber ser» como la imputación periférica de la que habla, el «no deber ser» carece de sentido o es una imputación negativa, lo que no es representable mentalmente. Todo el mundo tiene claro que una norma priva de validez a otra norma y ALCHOURRÓN y BULYGIN hacen consideraciones interesantes al respecto, dependiendo de si se sostiene una concepción hilética o una concepción expresiva de las normas. Creo que los problemas y su solución son más sencillos.

KELSEN ha observado que en algunas ocasiones los ámbitos de validez de la norma se encuentran indeterminados, como acontece cuando una norma establece la obligación de una persona moral o colectiva o cuando en un tratado se establecen obligaciones para un Estado. KELSEN dice que en estos casos, la indeterminación del ámbito personal de validez de la norma se elimina recurriendo al orden normativo constitutivo de la persona moral o del Estado, en el que se encuentra el sujeto individual que ha de realizar el acto obligatorio imputable centralmente a la persona moral o al Estado. Análogamente, en el caso de la derogación, encontré una indeterminación del ámbito temporal de validez de la norma, consistente en que se ha especificado la fecha inicial de vigencia, pero, en la mayoría de los casos, no se determina la fecha final de la validez de la norma. Las leyes, en sus artículos transitorios, establecen la fecha inicial de vigencia o validez de la ley, pero nada dicen respecto de la fecha final de su validez, es decir, del tiempo de su extinción. Creo que la derogación es la función jurídica que determina esta fecha final de validez de la norma en cuestión. La norma derogatoria no es una norma completa; es parte de la norma que deroga, creada en una fecha posterior. No hay porqué postular un «no deber ser», como una peculiar imputación negativa. También KELSEN dice que la norma derogatoria se refiere a otra norma para privarla de validez, pero es una norma que no regula conducta humana. Mi tesis evita este tipo de consideración, pues resulta entonces que hay normas que no regulan la conducta del hombre en sentido alguno.

Esta tesis mía también me explica la función de los órganos encargados de controlar la constitucionalidad o en general, la regularidad de un acto o una norma. En el caso del control de la constitucionalidad de una ley, por ejemplo, el órgano facultado para hacerlo, establece la fecha final de validez de la ley si determina como condición de ello su inconstitucionalidad. La nulidad de la ley se produce o significa que se ha determinado o establecido la fecha final de su validez cuando se ha constituido jurídicamente su irregularidad.

R.T.S.: Es bueno tener el resumen de tu tesis para reflexionar sobre ella y ver hasta qué grado me es aceptable. Esta tesis tuya de la derogación se conecta externamente con el concepto de la facultad. ¿En qué forma se lleva a cabo esta conexión?

U.S.O.: Creo que en gran medida coincidimos tú y yo en relación con el concepto de la facultad. Yo creo que es el concepto central de la jurisprudencia, incluso tan esencial como el de la coactividad para caracterizar al orden jurídico. La coactividad del Derecho se deja subsumir en el concepto de la facultad, convirtiéndose en uno de los po- 
sibles contenidos de ella, pues las sanciones impuestas por los jueces y demás órganos estatales, son el contenido de facultades otorgadas por normas jurídicas positivas.

Considero que la facultad es el contenido de las normas positivas que establecen los actos de producción de otras normas y determinan su contenido. La norma positiva que establece la facultad de un órgano para emitir una norma o realizar un acto, ha de establecer al mismo tiempo todas las dimensiones de realización de los actos integrantes del proceso de creación de la norma inferior. Por tanto, la norma que establece la facultad ha de determinar el o los sujetos que realizarán cada uno de los actos del proceso, es decir, el órgano de creación de la norma, el tiempo y el espacio de realización de los actos respectivos y el contenido de todos y cada uno de dichos actos, que es la dimensión material de realización de cada uno de los actos respectivos. Esta dimensión material de realización de los actos integrantes del proceso de creación de una norma inferior es de gran importancia, pues es el sentido o significación de esos actos. La norma creada es el significado de dicho proceso. Como puedes ver, este concepto coincide plenamente con el expresado por KELSEN. Es muy importante resaltar la parte final de esta definición.

Una última observación sobre este tema. Dije que la facultad es un contenido específico de normas positivas. Esto es muy importante, por dos razones: si el objeto de estudio de la jurisprudencia son las normas positivas, entonces la facultad tiene que ser un contenido específico de esta clase de normas. No puede ser de otra manera, una vez que hemos tomado la decisión metodológica de concentrar nuestros esfuerzos explicativos a esclarecer el concepto y el funcionamiento de los órdenes jurídicos positivos. Pero tiene una implicación adicional: la reformulación de la Norma Fundante Básica de KELSEN, por un Principio Fundamental de la Jurisprudencia. Si la Norma Fundante Básica es, según afirmación expresa de KELSEN, un presupuesto lógico-trascendental en el sentido de la filosofía kantiana, entonces no puede ser una norma, aunque sea ficticia, pues entonces se incurriría en una hipóstasis del presupuesto trascendental, al convertirlo en un elemento del objeto de estudio de la ciencia. KELSEN nos ha enseñado a prescindir y desenmascarar las hipóstasis, enseñanza que debemos aplicar ahora a su teoría de la Norma Fundante Básica. Lo que pasa es que el Principio Fundamental de la Jurisprudencia se proyecta sobre los hechos empíricos de la experiencia jurídica y de manera teórica se determina que lo que ha hecho el órgano constituyente es la creación de una constitución positiva, con lo que se posibilita la aplicación de todos los conceptos relacionados e implicados por el concepto de la facultad. Ciertamente, la postulación de una Norma Fundante Básica es un cómodo recurso expositivo, que tiene una simetría explicativa respecto de las normas positivas que establecen facultades. Es como si transitáramos de nuevo por un camino conocido.

U.S.O.: ¿Crees que hay coincidencia entre nuestras tesis sobre el concepto de la facultad?

R.T.S.: Creo que fundamentalmente estamos de acuerdo. Siguiendo de cerca las tesis de KELSEN puedo decir que la facultad describe una función fundamental del orden jurídico: precisamente la función de atribución o facultamiento de poderes (Ermächtigung), necesaria para la creación y aplicación del orden jurídico² ; fundamental para en-

2 Teoría pura del Derecho, Teoría pura del Derecho, UNAM, Instituto de Investigaciones Jurídicas/Editorial Porrúa, 1997 (reimpresión de la edición de la UNAM, Instituto de Investigaciones Jurídicas de 1979, pp. 157-159). 
tender el carácter dinámico del Derecho. Estas facultades son poderes específicos conferidos por el orden jurídico a ciertos individuos para participar en la creación del orden jurídico. En todos los casos públicos (jurisdiccionales o administrativos) o privados nos encontramos en presencia de una habilitación para crear normas jurídicas positivas.

El Derecho no es sólo un conjunto estático de normas de normas, sino es un «continuum de actos humanos que crean y aplican normas jurídicas». Esta concepción dinámica no siempre es hecha explícita. Sin embargo es siempre presupuesta en el trabajo cotidiano de la profesión.

Los actos que crean el Derecho ocurren en diferentes momentos y en gran variedad de cadencias. No obstante, sus entidades, i.e. normas (y los actos que las crean y aplican), son interdependientes entre sí. Las entidades de un orden jurídico se encuentran relacionadas de tal forma que para que el orden jurídico opere es necesario que los actos que crean y aplican las normas se realicen en una secuencia que va de los actos jurídicos condicionantes (facultamientos) a los actos condicionados (aplicadores), conexión sin la cual la creación jurídica «escalonada» no es posible ${ }^{3}$.

Es fácil percibir que el concepto clave en esta secuencia es el de facultad. «Facultad» nombra la función que, inter alia, permite identificar (i.e. determinar) las actos y normas subsecuentes. Ahora bien, si los actos de aplicación están condicionados por otros, entonces estos actos pueden ser representados como una secuencia de facultamientos (sit venia verba) de actos jurídicos ${ }^{4}$.

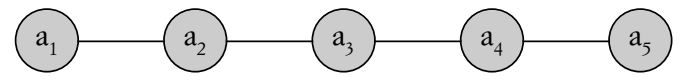

Donde los actos $a_{1}$ son condición de los actos $a_{2}$; y éstos condición de $a_{3}$, et sit cetera. No es difícil percatarse de que $a_{5}$ no condiciona ningún acto ulterior y, por tanto, es considerado las consecuencias de esta secuencia. A su vez, $a_{1}$, la condición más mediata de las consecuencias, tiene la particularidad de no estar condicionada por ningún acto.

Los actos jurídicos crean y aplican normas. Aunque las normas no son sino el contenido de los actos jurídicos (que los crean), es posible representar el aspecto fáctico del Derecho conjuntamente con su aspecto dinámico. Esto me obliga a introducir más elementos en el diagrama:

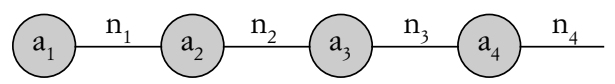

${ }^{3}$ Cfr.: Teoría pura del Derecho, pp. 232 y ss. En gran medida ésta es una reformulación de la tesis de KELSEN. Sobre la creación escalonada del orden jurídico, véase: OHLINGER, 1975: Der Stufenbau der Rechtsordnung. Rechtstheoriestiche und ideologische Aspekte, Viena: Manzsche Verlag- und Universitätsbuchhandlung.

${ }_{4}^{4}$ Joseph RAz llama a estas secuencias «cadenas de validez». En la construcción de estas secuencias de facultamiento he tomado de The Concept of a Legal System varias ideas (véase: op. ul. cit., pp. 97-99). 
Cada línea de unión representa una norma ${ }^{5}$ que faculta (habilita, autoriza) el acto que la aplica y el cual, a su vez, crea las normas que siguen en la secuencia. Así, $n_{1}$ autoriza los actos $a_{2}$, cuya realización aplica $n_{1}$ y crea $n_{2}$, aparece la misma relación genética que permite determinar e identificar los actos y normas posteriores ${ }^{6}$.

R.T.S.: Ulises, de todos es sabido que la casi totalidad de juristas sostienen que en las constituciones de los Estados se establecen derechos fundamentales (también les llaman «derechos humanos»). ¿Estás de acuerdo con esto?

U.S.O.: Sí y no. Así como el concepto de persona es un cómodo recurso conceptual para expresar la unidad de una pluralidad de normas, sin tener que descender a los pormenores de las relaciones, derechos y obligaciones unificados, de igual manera creo que hablar de «derechos fundamentales» es una cómoda expresión para referirse a una situación jurídica que no se especifica ni se describe, que se encuentra en la base de esas afirmaciones.

Mira, Rolando, es sabido que el concepto del derecho subjetivo fue elaborado tomando en consideración los intereses de los sujetos sometidos a un orden de normas que exigen cumplimiento y ejecución. La pregunta central sería entonces para ese sujeto: ¿’en qué me beneficia o perjudica, cuál es mi situación respecto de ese orden normativo coactivo que me exige obediencia y ejecución? En especial se hace énfasis en el beneficio que se puede obtener de ese conjunto de normas, lo que significa que la pregunta se transforme en esta otra: ¿cuándo puedo considerar ese conjunto de normas como «mi» derecho, como un Derecho que está a mi disposición y me beneficia? Los elementos que se consideran como respuestas a esta pregunta son la conducta lícita, los llamados derechos personales y reales, las autorizaciones y el poder o facultad. Salvo el último caso, todos los demás se dejan explicar por otros conceptos jurídicos distintos del «derecho subjetivo». La conducta lícita (o la licitud) es simplemente la ausencia de una norma que considera una cierta conducta como prohibida o lo que es lo mismo, la contraria como obligatoria. Respecto de una cierta conducta soy libre en relación con las normas del Derecho, que no la regulan en ningún sentido. HoBBEs hablaría de la libertad como derecho. Ese derecho es «el reflejo subalterno» de la no existencia de normas que regulen la conducta. El caso de los derechos reales y personales se deja explicar con el concepto de obligación, en un caso de todos (erga omnes) y en otro de sujetos determinados e individuales. Son nuevamente el reflejo subalterno de las normas que obligan a todos a abstenerse de intervenir en ciertas cosas (derechos reales) o a sujetos determinados a realizar ciertas prestaciones a favor de ciertos individuos (derechos personales). Las autorizaciones, tal como yo utilizo esta expresión, se dejan explicar con el

\footnotetext{
${ }^{5}$ «Norma» en el sentido de norma «unselbständige Rechtsnormen» («normas no independientes») (véase: Kelsen, H.: Reine Rechtslehre, cit., pp. 55 y ss. véase: Teoría pura del Derecho, pp. 68-70). Para KeLSEN las normas propiamente hablando se componen de toda la secuencia (cfr. ibid.).

${ }^{6}$ «La relación entre una grada superior y una inferior en un orden jurídico - como entre una constitución y una ley o entre una ley y una decisión judicial— es una relación de determinación... [L]a norma superior regula el acto por medio del cual la norma inferior es creada (o simplemente regula la ejecución del acto de coacción... Al regular la creación de la norma inferior, la norma superior determina no solo el proceso mediante el cual la norma inferior ha de ser creada sino, posiblemente el contenido de la misma» (KELSEN: Reine Rechtslebre [1. Auflage] pp. 90-91; véase: id., La teoría pura del Derecho. Introducción a la problemática científica del Derecho, cit., p. 127; id., Introduction to the Problems of Legal Theory, cit., pp. 77-78; id., Reine Rechtslebre [2. Auflage], pp. 346-349; véase: Teoría pura del Derecho, cit., pp. 349-351).
} 
concepto de la prohibición general y su restricción o levantamiento a un sujeto en particular si ha demostrado ciertas condiciones. Está prohibido de manera general manejar automóviles o herir a las personas a menos que se obtenga una licencia de conducir al haber demostrado ante la autoridad mi pericia en el manejo de esos artefactos o de haber cursado y sido entrenado en una escuela de medicina. El concepto central es el concepto de prohibición general y su restricción o levantamiento a ciertas personas. Hablar de «derechos subjetivos» en estos casos es una forma de presentar las funciones jurídicas mencionadas desde el punto de vista de los intereses de los sujetos sometidos a las normas.

Una función completamente diferente y que no se deja explicar con otros conceptos fundamentales es el poder o facultad. Ya te dije lo que entiendo por facultad: el contenido de normas positivas que establece el acto o actos (proceso) de creación o producción de otras normas y determina su contenido. Si hacemos el mismo tipo de razonamiento utilizado en los casos anteriores, donde se habla de derechos subjetivos, respecto de las normas que establecen facultades, los llamados «derechos fundamentales» no son otra cosa que los reflejos subalternos de las normas que establecen facultades. Para entender cabalmente este concepto hay que exponer en detalle los elementos de las facultades que no son otros que la serie de actos que componen el proceso de creación de la norma, $a_{1}, a_{2}, a_{3} \ldots a_{n}$ especificando las dimensiones personales, materiales, temporales y espaciales de cada uno de esos actos y relacionar esos elementos con los intereses de los sujetos a los que va a afectar la norma creada por ese proceso de producción normativa.

U.S.O.: ¿Cuál es tu postura en cuanto la obra de H. L. A. HART y de la anaylitical jurisprudence, en particular sobre tu postura sobre Joseph RAZ y, en virtud que tu traducción y tu larga estancia en Oxford, te acusan de un ortodoxo de las ideas de RAZ sobre razones.

R.T.S.: Ciertamente mucho me honra y dignifica que me asocien con la Universidad de Oxford; muchas cosas aprendí y sigo aprendiendo de HART; Pero guardo cierta distancia con respecto a varias de sus ideas. Ulises te contesto primeramente la última parte de tu pregunta porque mi relación académica con Joseph fue anterior.

U.S.O.: Bueno, ¿cuál es tu postura con respecto a RAZ y, en especial, con su teoría de razones?

R.T.S.: Mi primera aproximación hacia el trabajo de RAZ — quizás no lo creas—fue muy positiva y sugerente (entonces RAz hacia filosofía del Derecho). A la sazón, yo escribía mi libro Sobre el sistema jurídico y su creación ${ }^{7}$. Juan REBOLLEDO (colega y amigo), quien sabía de lo que yo me ocupaba, en viaje en Inglaterra, halló el libro de RAz: The Concept of a Legal System ${ }^{8}$ y me lo obsequió. Juan observó que desde el título (o mejor el subtítulo) existían ciertas semejanzas, alguna similitud en su estructura, conjunto de temas y un parecido en las gráficas que al menos, perseguían los mismos propósitos. El libro de RAZ era notablemente superior al mío, en redacción, datos y simbología. Este libro fue, en principio su tesis que dirigió el profesor H. L. A. HART. El

\footnotetext{
${ }^{7}$ México: UNAM, Sobre el sistema jurídico y su creación, México: UNAM. Instituto de Investigaciones Jurídicas, 1976 (Serie G: Estudios Doctrinales, n. ${ }^{\circ} 16$.

${ }^{8}$ Oxford: Oxford University, at Clarendon Press, 1970.
} 
libro me pareció un buen libro y lo traduje, como lo hizo el amigo y colega Paolo ComMANDUCI.

Durante el proceso de traducción, encontré muchos aciertos. La simple inclusión de Austin y de Bentham es, sin duda un acierto. Aciertos también lo son la introducción de los criterios de identidad y de existencia de los órdenes jurídicos y el tratamiento de la estructura e individuación de las normas. Hace tiempo (no sé cuanto) participé en un Seminario dirigido por el «jefe» Ernesto GARZÓN, donde expuso con detalle y precisión las Lectures of Jurisprudence de John AusTiN. Con ese conocimiento caí en cuenta de que el libro de Joseph no hacía justicia a John Austin.

Un desacierto es el tratamiento simplista con el que RAZ exhibe a Austin. El profesor de Londres es mostrado como un jurista inocentón que sostiene: «el derecho es un "mandato" » (con gran asombro de los juristas formados en la idea de que puede haber derechos antes o por fuera de un orden jurídico). RAZ procura mostrar los defectos del mandato, ignorando que son muchas las cuestiones que muestran que sus Lectures of Jurisprudence son una obra magistral; muy completa y con gran economía de conceptos.

El tratamiento de la obra de KELSEN no es mejor. Particularmente se distorsiona la complejidad y funcionamiento del orden jurídico que proporciona la teoría de KELSEN al someterlo a un esquema notablemente simple. RAZ sostiene que el concepto de la norma fundamental no satisface los criterios de identidad ni de unidad del orden jurídico, en la medida que uno la asume. RAZ pasa por alto un ejemplo muy ilustrativo de KELSEN. Supóngase que en una reunión de talentosos abogados que confeccionan un difícil contrato, que de repente, saltara uno o dos preguntando: «si el código y demás leyes que estaban aplicando eran válidas». Es claro que los abogados partícipes asumen, sin lugar a dudas, que lo que hacen es válido y tienen como válido el código que aplican. Es en virtud de que se asume que hay constituciones y códigos válidos.

Ahora bien, Ulises, en cuanto a mi postura sobre la tesis de Joseph de que las normas son razones. Creo que mi postura quedó manifiesta desde el XVIII Congreso Mundial de la IVR en mi ponencia «How to make Reason with Norms. Or How to Convert Norms Into Reasons.; or How Disentangle this Mess». A petición del profesor y amigo Manuel ATIENZA hice una versión en español'.

Regreso al hecho positivo de que varios aspectos de la teoría de John AustiN son tratados por RAz. También introduce el concepto de «cadena de validez» con la que pretende dar cuenta de algunos problemas de la Stufenbautheorie. La introducción de la arborecencia sustituye con ventaja la idea de una estructura piramidal. La idea de la arborecencia, la tomé de RAZ (aunque la norma jurídica independiente ya había sido descrita por Carlos ALChOURRÓN años antes). La diferencia con RAZ es que introduzco en esta arborecencia el concepto de facultad como «un facultamiento [condicionamiento] sucesivo» en la creación del orden jurídico. Este nuevo esquema de arborecencia empezó con mi libro El Derecho y la ciencia del Derecho (Introducción a la ciencia

\footnotetext{
9 ¿Cómo hacer razones con normas, o cómo convertir normas en razones o cómo desenredar este lío» (Doxa, Cuadernos de Filosofía del Derecho, vol. II, n. ${ }^{\circ}$ 21, 1998, Actas del XVIII Congreso Mundial de Filosofía del Derecho, Alicante, España: Universidad de Alicante, pp. 437-462 (junio 1999).
} 
jurídica ${ }^{10}$. Y continúo manteniéndola en la última reimpresión de Elementos para una teoría del Derecho ${ }^{11}$ (3. ${ }^{a}$ reimpresión, de la 2. ${ }^{a}$ edición).

En cuanto a las tesis de RAZ sobre las razones y su división entre razones de primer orden y razones excluyentes y particularmente su tesis de que las normas jurídicas son razones, y si esa es su característica definitoria, la rechazo enfáticamente.

Movidos por la fascinación que irradia la argumentación y el razonamiento jurídicos, algunos autores han llegado al extremo de caracterizar a las normas jurídicas como razones (e incluso convertir al Derecho en mera argumentación). Al hacerlo cometen un tipo de falacia naturalista. Las normas (como cualquier cosa) pueden ser tratadas como razones (justificaciones) para la acción, pero es un error que por ese hecho se pueda pensar que las normas sean sólo razones.

Para los defensores de esta tesis cualquier cosa puede ser una razón. Llover es una razón para usar una gabardina. El problema es que no todo necesariamente es una razón para actuar. Que llueva en Kuala Lumpur, no es razón para usar gabardina, toda vez que me encuentro en México. ¿Qué convierte a un hecho en una razón?

No veo otra respuesta, que la siguiente:

$x$ es una razón si, y sólo si, el agente la toma en cuenta para actuar o para abstenerme de actuar.

Piénsese, por ejemplo, en algo que ignoro ¿Cómo podría ser una razón para mi acción? Ciertamente, si en mi deliberación tomo en consideración un hecho $X$ (sea acontecimiento o dictum), entonces éste se convierte en una razón para mi acción.

U.S.O.: Rolando, hablas en tu ensayo de una «regla de reconocimiento de razones». ¿Esta es una aplicación de la teoría de HART de la regla de reconocimiento para refutar a RAZ?

R.T.S.: A las condiciones que deben satisfacerse para que cierto hecho se convierta en una razón la llamo: «la regla de reconocimiento de razones». Esta regla no es mas que un criterio de identidad de razones. En breve: se puede decir que algo es una razón si, y sólo si, es reconocida por el agente.

Si cualquier cosa puede ser una razón, entonces, sin duda, las normas (jurídicas), pueden ser razones. Pero, para que una norma sea una razón, tiene que satisfacer la regla de reconocimiento de razones. De esta manera, si yo no tomo en consideración a la norma $N$ entre las razones para hacer $\varphi$ (o para omitir $\varphi$ ), entonces dicha norma $N$ definitivamente no es una razón para que yo haga u omita $\varphi$. Y, si no obstante, la norma $N$ se me aplica, este es un «hecho que ocurre» (un hecho que me pasa»), no una acción que yo hago.

De lo que anteriormente he dicho se sigue que existen dos diferentes criterios de identidad para dos diferentes clases de cosas.

Uno: Un hecho $x$ se convierte en una razón si, y sólo si, el agente, reconoce a $x$ como razón para actuar o para abstenerse de actuar.

\footnotetext{
${ }^{10}$ México: UNAM. Instituto de Investigaciones Jurídicas, 1984 (ISBN 968-837-366-4).

${ }^{11}$ México: Editorial Themis, 3. ${ }^{a}$ reimpresión de la 2. ${ }^{a}$ ed., 2005 (ISBN 968454621 1).
} 
Dos: Un hecho $\mathbf{x}$ es $N$ (una norma jurídica) si, y sólo si, $N$ es creada por una instancia creadora del Derecho i.e., por el legislador L (latissimo sensu).

Esto significa que las normas, per se, no son razones, aunque pueden funcionar como tales en cierto contexto.

Que yo reconozca normas jurídicas como razones de mi acción no hace que mi reconocimiento se convierta en característica definitoria de las normas jurídicas. Puedo «entender» a las normas jurídicas como «motivos», «causas», «estímulos» o «razones», pero las normas jurídicas no son normas jurídicas por ser «motivos», «causas», «razones»; son normas jurídicas porque satisfacen la regla de reconocimiento de normas.

Recordemos rápidamente la objeción de ignorantia iuris. Las normas (jurídicas) funcionan como normas, aun cuando el agente no las conozca. Por el contrario, las normas jurídicas son razones porque el agente las convierte en razones. El agente adopta razones, cierto; pero, para que las normas jurídicas existan y se apliquen, no requieren ser adoptadas como razones por los destinatarios. Las normas jurídicas se aplican ex auctoritas y su ignorancia no excusa a nadie de su incumplimiento.

Si se puede perfectamente describir una norma (y los hechos sociales que la establecen) sin recurrir al concepto de razón para actuar, entonces las normas jurídicas y las razones para actuar son conceptos distintos y separados. Esa podría ser una individuación de «la tijera de Bentham».

Si el agente actúa sobre la base de $N$, entonces $N$ es una razón que «vence» otras razones, y esto significa que habiendo sido considerada por el agente como una razón, una razón exitosa, una razón que supera otras razones. Por el contrario, si el agente, habiendo convertido a $N$ en razón y ésta no es exitosa, ésto sólo significa que $N$ ha sido superada por otras razones.

Los defensores de las razones para actuar afirman que éstas pueden ocupar (al menos) dos diferentes niveles: Pueden ser (1) razones de primer orden u ordinarias o bien, (2) razones de segundo orden. Las razones de primer orden (u ordinarias) son razones para la acción o para abstenerse de la acción. Por el contrario, las razones de segundo orden no son razones para la acción (inmediata), sino razones para seleccionar o excluir razones para la acción.

Joseph RAZ da el siguiente ejemplo. Un padre le dice a su hijo: «compórtate como diga tu madre». Esta instrucción es una razón para actuar por ciertas razones: las instrucciones de su madre, las cuales, son también, razones. Supóngase ahora que el padre le dice a su hijo: «no te comportes como diga tu madre». En este caso, el hijo tiene una razón para no actuar por ciertas razones. RAZ llama a las razones de segundo orden negativas: «razones excluyentes» ${ }^{12}$.

Una razón excluyente requiere que el agente ignore clases de razones. Los autores — de este orden de ideas - piensan que este es un rasgo característico de las normas jurídicas. Regresemos al ejemplo: Esta es una razón de segundo orden sólo asumiendo

${ }_{12}$ The Authority of Law. Essays on Law and Morality, Oxford: Oxford University Press, 1979, pp. 16-17 (existe traducción mía: La autoridad del Derecho. Ensayos sobre Derecho y moral, México: UNAM, Instituto de Investigaciones jurídicas, 1982, pp. 31-32). 
que la orden del padre haya sido considerada por Juan (el agente), primero, como una razón y, además, como razón excluyente.

U.S.O.: Las normas jurídicas no son ni pueden ser razones excluyentes, porque no establecen que deban desconocerse otras razones para actuar o abstenerse de actuar. Que puedan llegar a considerarse «razones excluyentes» puede derivar del hecho de que han sido establecidas por una autoridad u órgano jurídico y que, además, puede aplicarse al sujeto un acto coactivo en caso de incumplimiento.

R.T.S.: Sí, el carácter excluyente tiene que ser reconocido por el agente. Pero, si considero a las normas como razones, éstas no necesariamente se convierten en razones de segundo orden excluyentes. Ciertamente, puedo considerar a las normas jurídicas como razones excluyentes. Pero, sólo lo puedo hacer cuando al ser consideradas en mi deliberación para actuar, las acepte como razones de segundo orden que me requieren que ignore todas las razones que estas razones excluyen. Si es así, resulta claro que esto funciona porque yo (el agente) lo hago, no porque sea cualidad intrínseca de las normas jurídicas ser razones y, menos, necesariamente excluyentes.

Veamos una versión un tanto diferente («el caso del tazón de avena»). Supongamos una instrucción del padre que dice: «Juan actúa siempre como dice tu madre y no tomes en cuenta ninguna otra razón que pudieras tener». El espectro de posibilidades es el siguiente: 1) Juan puede ignorar totalmente la instrucción de su padre. 2) Juan puede considerar la instrucción de su padre como razón ordinaria. 3) Juan puede adoptar la instrucción de su padre, reconociéndola como razón y considerarla, como razón excluyente.

Durante cierto tiempo Juan considera la orden de su padre como una razón excluyente y siempre que su madre ordena algo actúa en base a lo que ella dice, ignorando cualquier razón en contra que él pudiera tener. Pero, un buen día, la madre de Juan le dice que ingiera un tazón de avena. Esto fue el límite. A partir de entonces, Juan decide desobedecer sistemáticamente; desde entonces actúa en base a sus propias razones para no comer avena: la avena se ve horrible, huele horrible y sabe horrible.

Desde que Juan empezó a actuar en base a sus propias razones, la instrucción de su padre, eo ipso, perdió su carácter de razón excluyente. No fue fácil para Juan desobedecer a su madre adorable, aunque fue más difícil desobedecer a su estricto y severo padre (Juan sabe muy bien las consecuencias que esta desobediencia le acarrea). Sin embargo, desde ese día, Juan no comió más avena; sin importar lo que pudiera venirle.

Según Raz las normas jurídicas son razones excluyentes y si pierden este carácter pierden, también, el carácter de normas. Si una razón no es una razón excluyente, no es una norma ${ }^{13}$. Pero ¿esto es así? Supongamos que le preguntamos a Juan. ¿Crees que la orden de tu padre ha desaparecido? ¿que ya no existe? ¡Por supuesto que no! La orden existe; es precisamente la orden que Juan desobedece.

Es realmente extraño, que en el caso de nuestro ejemplo, el rasgo característico de las normas - como afirman los defensores de esta tesis - pueda ser eliminado y que, sin embargo, continúen siendo normas. Esto recuerda la objeción de ignorantia

${ }^{13}$ E.g.: el propio Joseph RAZ (cfr.: ibid.). 
iuris: en donde su ocurrencia revela que las normas son normas, aun cuando no sean consideradas razones excluyentes o, incluso aunque no sean consideradas razones en absoluto.

Lo que pasa con Juan y con su padre pasa igual con una norma jurídica, por ejemplo, con una resolución de un juez. Simplemente supóngase que la instrucción del padre es una decisión judicial que ordena a la parte convicta a pagar una suma de dinero (y que ignore, $a b o b v o$, cualquier otra razón que pudiera tener). El espectro de las posibilidades de la parte perdedora es exactamente igual al que enfrenta Juan, a saber: 1) Puede ignorar por completo la sentencia (y volverse recalcitrante como lo fue Juan). 2) Puede considerar la decisión del juez, pero sólo como razón ordinaria, considerarla pero esta razón puede ser vencida por otras razones que pudiera tener. 3) El convicto es un capo y decide, por razones prudenciales, someterse al Derecho.

Joseph RAz proporciona otros ejemplos los cuales, de alguna manera, contrastan con el que he analizado anteriormente. Sólo haré un comentario sobre el caso Jeremías.

Jeremías es un soldado. Su superior le ordena tomar un vehículo que pertenece a un civil. Jeremías, en el caso que analizamos, está consciente de que tomar el vehículo es un exceso y conoce mejores formas para lograr lo que su superior quiere. Sin embargo, Jeremías es un buen soldado y, no obstante sus propias consideraciones, recuerda que «...órdenes son órdenes y deben ser obedecidas aún sean equivocadas» ${ }^{14}$.

La noción de razón excluyente es claramente descrita en el ejemplo. Sin duda podemos distinguir la orden de un superior que funciona excluyendo todas las razones, excepto ella misma.

Este caso difiere del caso de Juan y su padre. En este caso existen dos instrucciones diferentes. La primera, la del padre y, la segunda, la de la madre. Consecuentemente, tenemos dos tipos de instrucciones, emitidas, incluso, por dos diferentes sujetos, en dos diferentes momentos. Si ambas instrucciones (que son dos hechos completamente diferentes) satisfacen la regla de reconocimiento de razones, ambas funcionan como razones para el agente. En el caso del soldado (como en los otros dos ejemplos), no hay dos actos de emisión de instrucciones: la orden del superior constituye una sola y única razón que el agente tiene que enfrentar (Una razón externa a las razones que tienen que ser pasadas por alto o excluidas). RAZ señala que «... el mismo hecho... es, al mismo tiempo, una razón para la acción y una razón [excluyente] para pasar por alto las razones en su contra» ${ }^{15}$. De esta peculiar opinión resulta un lío.

En el caso Jeremías dos aspectos entran en juego. El primero, la orden dictada por el superior: «soldado tome el vehículo»; el segundo, el hecho de que uno tiene que pasar por alto todas las otras razones (de primer orden) en contra. Lo difícil de aceptar de esta tesis es que este último aspecto se encuentre inserto en la orden «soldado tome el vehículo» dada por el superior.

En el caso de Jeremías no es problema separar los dos niveles de razones. Uno: la orden dada por el superior y la proposición: «las órdenes (militares) deben ser obedecidas sin considerar sus propios méritos» (una razón de segundo orden). Esta última

${ }_{14}$ Cfr.: Practical Reason and Norms, cit., p. 38 (Razón práctica y normas, cit., p. 43).

${ }^{15}$ The Authority of Law, cit., p. 18 (La autoridad del Derecho, cit., p. 33). 
razón, sin duda, es parte de la situación peculiar que hay que destacar: Jeremías es soldado. Si no fuera así, ¿de dónde hubiera sacado Jeremías la idea de que «las órdenes son órdenes» y que él tiene que obedecerlas?

La orden del superior podría haber sido ciertamente ignorada si Jeremías, no siendo soldado, hubiera podido desentenderse de la situación. Pero, siendo soldado, Jeremías ni siquiera puede ponderar otras razones para decidir; éstas no fueron consideradas por Jeremías porque Jeremías, previamente, adoptó otra norma (una Grundnorm): «como soldado debo obedecer las órdenes (militares) sin considerar sus propios méritos». Ésta es una razón que deviene fundamental la cual, desde que la adopta Jeremías, gobierna su comportamiento como soldado.

Esto es así desde que Jeremías decide jugar el juego del soldado ${ }^{16}$. El dicho «órdenes son órdenes...» interviene decididamente en la consideración de Jeremías porque es una consecuencia que se sigue de jugar al soldado.

U.S.O.: Rolando, vuelvo sobre la pregunta anterior. Tu hablas de «jugar con las reglas del juego». ¿No es esto una forma de expresar la autoridad y la validez objetiva del Derecho? La metáfora del juego es muy ilustrativa, pero puede prescindirse de ella.

El uso de la expresión «juego» en esta última frase no pretende ser una exagerada metáfora (pensemos en el drama griego y en el papel dramático que hace un individuo en la vida social). Más bien su uso tiene la intención de mostrar qué tanto el juego nos permite entender la aplicación de normas (u otras directivas) ${ }^{17}$.

Desde antiguo «juego» (del L. jocus) y sus equivalentes nombra un conjunto de acciones con sujeción a reglas (donde los participantes se esfuerzan por ganar). El uso habitual de la expresión «las reglas del juego» sugiere la idea, prácticamente indisputable, de que los juegos tienen reglas. Varios autores han explicado con éxito cómo funcionan las reglas de los juegos ${ }^{18}$.

El juego es un universo limitado: El juego sólo se juega con jugadas del juego. De hecho, esta sería algo así como la Grundnorm o, mejor, la Grundregel (i.e. «regla fundamental») de los juegos. Pues bien, es precisamente la adopción de esta Grundregel lo que nos hace excluir todo aquello que no es jugada del juego.

Volvamos al «juego del soldado». Jeremías enfrenta dos diferentes situaciones en tiempo. La primera, la decisión de «jugar al soldado»; la segunda, la orden del superior $^{19}$. Jeremías obedecerá las órdenes de su superior mientras continúe «jugando al soldado». Jeremías puede abandonar (él puede desertar e, incluso, resistir a la autoridad).

${ }^{16}$ Puede ocurrir que la decisión de «jugar al soldado» no sea siempre completamente «libre», como sucede en los casos de reclutamiento forzoso.

${ }^{17}$ No me puedo detener aquí para hablar de esto, pero más bien el juego puede ser considerado como una metáfora de la vida social. El juego es como el drama y la comedia, una forma de representar la vida.

${ }^{18}$ Un buen ejemplo es el excelente libro de Robles Morchón, G., 1988: Las reglas del Derecho y las reglas de los juegos. Ensayo de teoría analítica del Derecho, México: UNAM; Instituto de Investigaciones jurídicas, Serie G, Estudios Doctrinales, n. ${ }^{\circ}$ 14).

${ }_{19}$ Aunque estas dos diferentes situaciones se dan generalmente en dos diferentes momentos, puede ocurrir que estos dos momentos se presenten prácticamente de forma simultánea. Como podría ser el caso de una adhesión a un líder a quien se sigue de inmediato. Sin embargo, es fácil observar que la decisión de «tener a alguien por su líder» y obedecer sus órdenes son dos hechos totalmente distintos. Como lo son «jugar al soldado» y las órdenes de sus superiores. 
Este «juego» es fácil de jugar (en el sentido de que es fácil entender cómo se juega). Como cualquier otro juego, este juego se juega realizando «jugadas del juego», i.e. obedeciendo las órdenes militares superiores.

En contraposición con esto, RAZ considera la orden del superior (una sola razón) como si fuera al mismo tiempo una razón de segundo orden y una razón de primer orden.

No cabe duda que en casos como el de Jeremías es indisputable que la razón ordinaria presuponga la regla fundamental del juego ${ }^{20}$. Sin embargo, el hecho de que una razón presuponga a otra no es suficiente para tratarlas como si fueran una y la misma.

La construcción de esa «razón duplex» es realmente una entidad extraña. Pero, ¿Cómo podría una orden militar (i.e. una «jugada del juego castrense») obligar a quien no es soldado (para quien no juega ese juego)? Pero, aún peor ¿cómo es que la orden del superior pudiera establecer la Grundregel de un individuo que, como Jeremías, decide «jugar» al soldado?

¿Por qué convertimos normas en razones (para la acción)? La respuesta es simple: «para jugar juegos». Para jugar juegos usamos reglas (normas). Existen, sin embargo, actividades humanas que no se llaman «juegos» y que usan normas. Por ejemplo, el Derecho.

Voy a sostener la tesis de que la argumentación y, en particular, la argumentación jurídica, puede ser descrita en cercana analogía con jugar juegos. Al menos con el de Jeremías, cuando decide jugar al soldado.

Supongamos que el padre de los anteriores ejemplos decide jugar ajedrez con Juan, su hijo. El padre, irritado por el curso que ha tomado el juego decide mover su «rey» cinco cuadros de manera diagonal, arguyendo que si los «alfiles» lo pueden hacer, el «rey» no puede hacer menos. No obstante el «impecable» argumento del padre, Juan descalifica la acción diciendo que no es una jugada del juego. Más tarde, el padre de Juan objeta airadamente un «enroque» realizado por Juan, arguyendo que es injusto toda vez que él estaba atacando ciertos cuadros y, ahora, el «enroque» hace que sus jugadas sean completamente inútiles. A esto Juan contesta que el «enroque» es una jugada del juego. Después de esto, el padre de Juan pierde un peón por una captura «al paso». El padre, alegando que ignoraba que se pudiera «comer al paso» reclama la devolución de su peón. Juan dice que «comer al paso» es una jugada del juego y, por tanto, la jugada se mantiene. Manifiestamente incómodo el padre de Juan interrumpe el juego diciendo a su hijo: «estoy harto de tu estúpido estribillo: "jugada del juego". ¡ Vete a tu cuarto! Voy a continuar el juego con "jugadas" de mi propio juego. En esta casa soy yo quien decide qué es una "jugada del juego"». Sin embargo, el padre de Juan sabe perfectamente bien que esto no es verdad y que si quiere ganarle a su hijo un juego de ajedrez, la única manera de hacerlo es usando únicamente «jugadas del juego» y no con autoridad paterna.

Juan, prudentemente, se fue a su cuarto jugando el juego del «hijo obediente», sabiendo que el ajedrez, como cualquier otro juego, se juega exclusivamente con jugadas reconocidas como jugadas del juego.

${ }^{20}$ Una orden realmente primera, dada por el primer líder sobre la tierra, aunque sea una y misma orden, presupone una norma fundamental: «obedece al primer líder (y pasa por alto otras razones)». Ésta es la única forma de que alguien sea primer líder. Si algunos hombres le obedecen es porque han adoptado la norma fundamental: una norma que no fue establecida por el primer líder. 
La moraleja de este ejemplo es que cuando decidimos jugar juegos adoptamos la regla fundamental del juego la cual podría formularse como sigue:

Juega sólo con jugadas del juego y pasa por alto cualquier razón que puedas tener.

Las «jugadas del juego» son identificadas por las reglas que constituyen el juego (las reglas que lo gobiernan ${ }^{21}$. De esta forma, la regla fundamental del juego puede ser reformulada como sigue:

Al jugar juegos, actúa únicamente sobre la base del conjunto de reglas que identifican sus jugadas (y, ab obvo, ignora cualquier otra consideración)

Lo que los jugadores (quae jugadores) hacen con las reglas del ajedrez o con las reglas de cualquier otro juego, algunos individuos (en su mayoría, miembros de la profesión jurídica) lo hacen con las normas jurídicas cuando deciden jugar el juego de la «argumentación jurídica» y adoptan la correspondiente norma fundamental.

Es muy importante tener en mente que una cosa es considerar a las normas desde el punto de vista de un destinatario y muy otra jugar a «la argumentación jurídica». En este «juego» los jugadores tienen que pasar por alto todo tipo de razones salvo uno: el que constituye el punto de vista jurídico. El punto de vista jurídico es proporcionado por las normas jurídicas que gobiernan el «juego».

U.S.O.: Bueno, ahora dime tu postura con respeto a H. L. A. HART y en general a la analytical jurisprudence.

R.T.S.: Estimado Ulises, como te he dicho, mi posición con respeto a la analytical jurisprudence y la obra de HART es de respeto y admiración. La analytical jurisprudence dio ocasión a replantear preguntas y permitió a la teoría jurídica escaparse del fantasma que lo encubría: el lenguaje iusnaturalista y metafísico. Ciertamente, la analytical jurisprudence ha recibido muchos beneficios de la moderna filosofía analítica y del análisis del lenguaje llevado acabo por diferentes disciplinas. Sin embargo esta corriente del pensamiento jurídico se mantiene fuertemente ligada a sus orígenes. La analytical jurisprudence es una forma de positivismo jurídico y, como tal, comparte rasgos característicos de otras formas del positivismo.

Propiamente hablando el primer tratado sistemático de esta corriente del pensamiento jurídico se debe a John AusTin (1790 1859), el más preclaro jurista inglés del siglo XIX, aunque no minimizo la influencia que haya podido tener BENTHAM en su obra.

Ciertamente, el análisis del lenguaje en que el Derecho se formula y las operaciones intelectuales que realizan jueces y abogados es necesariamente un estudio de derecho positivo. Ulises en razón del espacio no puedo desarrollar tu pregunta de por qué hablo del modicum de derecho positivo presente en la dogmática jurídica y en los usos de la profesión. Basta señalar que cualquiera que sea la doctrina que se tenga, un tomista, un troskista, un anarquista y un positivista, cuando se trata de aplicar $v$. gr. lo tendrán que hacer de conformidad con las prácticas de la profesión. Tendrán primeramente que determinar el derecho aplicable para ello deberan probar que existe

\footnotetext{
${ }^{21}$ Sobre este particular, véase: RoBLES, 1988: Las reglas del Derecho y las reglas del juego, México: UNAM, Instituto de Investigaciones Jurídicas; SCHAUER, 1991: Playing by the Rules: A Philosophical Examination of the Rule-Based Decision-Making en Law and in Life, Oxford: Oxford University Press.
} 
(que ha sido puesto, que no ha sido derogado, que no hay caducidad, que no ha prescrito, ni precluido). Esto es, cuando hacen de juristas, su credo y sus ideologías tienen que dejarse de lado.

AUSTIN al analizar las nociones en uso en el Derecho inglés, delimitó el campo (the province) de la jurisprudencia, que no puede ser otro más que el derecho positivo. Para ello era preciso establecer un claro deslinde para delimitar el Derecho y trazar una línea divisoria entre el Derecho y la moral línea que, consideraba Austin, estaba oscurecida por las doctrinas del Derecho natural.

Ulises, tengo la firme convicción de que el núcleo de la analytical jurisprudence se encuentra en las Lecture de Austin. Obra que en su tiempo no fue entendida. AusTiN estableció los lineamientos básicos y los temas de la analytical jurisprudence cuyo hilo conductor es el significado de las palabras.

En cuanto a HART debo decir que su obra tiene varios méritos de los cuales mencionaré tres. Primeramente es excelente por precisa, clara y novedosa; en segundo lugar es oportuna porque aparece después del regreso de los fantasmas del Derecho natural de la posguerra. En tercer lugar, hace un estudio crítico de KELSEN y de la Teoría pura mostrando a un KELSEN ignorado por la literatura de lengua inglesa. Existe un mérito más, dialoga con John Austin y más importante quizás, desarrolla algunas ideas que Austin dejó claramente apuntadas. Aprecio el ingenio y talento de Austin.

Para HART el propósito general de la jurisprudencia analítica es lograr un mejor entendimiento del Derecho y para ello es necesario hacer un cuidadoso examen del lenguaje en que el Derecho se formula. Siguiendo a AUSTIN, HART aborda las nociones recurrentes del discurso jurídico.

Para quien el planeamiento general de la jurisprudencia estaba equivocado. En esta línea HART aborda al análisis de los conceptos jurídicos y sostiene que la manera habitual de formular definiciones de conceptos jurídicos es inadecuada ${ }^{22}$. Recuerdas Ulises que me preguntabas por qué decía que el tema de la definición de conceptos jurídicos que HART desarrolla en su Inaugural Lecture ya se encontraba en AusTIN. Pues bien la respuesta está en una frase genial de AusTin. Para John Austin el objeto de la jurisprudencia es la elucidación de los «principios, las nociones y las distinciones». Este giro es indispensable porque AUSTIN considera que algunos de sus términos no admiten una definición en la forma habitual; más aún definirlas en esa forma —insiste AustiN- es del todo inútil» ${ }^{23}$.

Teniendo como antecedente este atisbo genial de Austin, HART señala que cuando los juristas intentan definir esas palabras no se percatan que éstas no tienen una conexión directa con el universo observable. Estas palabras realizan una función específica en el marco del orden jurídico ${ }^{24}$.

${ }_{22}$ Véase «Definition and Theory in Jurisprudence», en HART: Essays in Jurisprudence and Philosophy, Oxford: Oxford University Press.

${ }^{23}$ Véase «The Uses of the Study of Jurisprudence», en The Province of Jurisprudence Determinbed and the Uses of the Study of Jurisprudence, HART, H. L. A. (ed.), Indianapolis Hackett Publishing Company Inc. [reimpresión de la edición de Weidenfeld \& Nicholson, Londres, 1971 (1954), pp. 370-371]. pp. 21-48.

${ }^{24}$ Véase, «Definion and Theory i jurisprudence», en HaRT: Essays, iin jurisprudence and Pbilosophy, cit., 
U.S.O.: Estoy de acuerdo con lo que dices respecto a AuSTIN. ¿'Te parecería correcto afirmar que HART reformula algunas, pocas, tesis de KELSEN y con ausencia de pulcritud conceptual, porque introduce términos sociológicos y psicológicos que están fuera de lugar en una teoría jurídica? Ejemplo, en vez de hablar de la coacción jurídica habla de «presión social considerable».

R.T.S.: En cuanto a HART, inmediatamente me adherí a su posición que expresa en Definition and Theory in Jurisprudence en así como a su postura en los debates con Leon FulLER y con Edgard BODENHEIMER. Creo que, sin embargo, mi posición sobre HART está infinitamente dividida. Tengo la convicción de que muchos de los aciertos de HART ya se encontraban en KELSEN. Como el propio HART dijo: «KELSEN es el más grande de los juristas analíticos». Creo que HART en cierta medida sólo hace una reformulación moderna de la Teoría pura. Pero, HART (muerto KelSEN) es sin duda el mejor jurista del siglo Xx y líder de la analytical jurisprudence contemporánea.

Encuentro que es un grave error sostener que el lenguaje en que el Derecho se formula es un lenguaje de «textura abierta», hecho que ha motivado muchos malentendidos. Es más simple sostener que cualquier conducta puede ser regulada por el Derecho si ésta ha sido establecida por las instancias creadoras del orden jurídico. Algunos autores han creído ver en esa expresión una fusión entre Derecho y moral; sin embargo, los alcances de esta idea son más limitados. Esto se aprecia fácilmente considerando el funcionamiento de la regla de reconocimiento. La regla de reconocimiento es un criterio de identidad que establece que un imperativo o una norma es Derecho inglés si los jueces ingleses (lato sensu) consideran que deben aplicarlo.

Que los jueces incorporan nuevo material al orden jurídico es un hecho indiscutible; pero esto no se debe a las características de la «textura» en que el Derecho se formula sino a hecho indiscutible que es creación del juez. El orden jurídico es un sistema creado por el órgano (u órganos) «habitualmente obedecidos». El orden jurídico sólo se compone por las disposiciones creadas por las instancias creadoras de Derecho.

Ciertamente existen procedimientos extraordinarios que modifican el sistema jurídico: la costumbre contra legem (en donde las disposiciones del órgano no son obedecidas, i.e. desuetudo) y la revolución (que produce la sustitución del sistema o, al menos la división del sistema). Pero como quiera que sea esto no es textura abierta sino actos de creación de las instancias habitualmente obedecidas.

Si bien entiendo que una norma jurídica independiente se forma de varios y actos condicionados de creación, HART es muy claro cuando sostiene que la profesión no piensa en una norma jurídica así. Por exacto que pudiera ser, como creo que lo es, la norma jurídica completa (como lo ha aceptado el mismo Robert WALTER) no satisface los requisitos meta teóricos de claridad y aparentemente distorsionan la visión de jueces y abogados. Creo que es muy inteligible la división que presenta HART entre Duty Imposing Rules y Power Confering Rules, y que proporciona claridad sobre sus diferentes funciones. Si bien HART conviene en que esas diferentes reglas tienen que estar relacionadas, no da cuenta de las funciones que realiza el concepto de facultad, en la creación del Derecho y aplicación del Derecho. Por ello la teoría de Raz no da cuenta de 
esas funciones ni del elemento más importante de las normas que facultan: mostrar el aspecto dinámico del Derecho.

Por su economía de conceptos, prefiero a AUSTIN; por la completitud de su teoría me inclino sencillamente por KELSEN.

U.S.O.: Con esto, estoy totalmente de acuerdo. 